\title{
ANÁLISIS DEL DISCURSO EVALUATIVO EN DOCENTES DE EDUCACIÓN FÍSICA
}

Analysis of the evaluative discourse in teachers of physical education

Análise do discurso de avaliação em professores de educação física

\section{Beatriz Elena Chaverra Fernández}

Profesora de la Universidad de Antioquia. Teléfono: +574 2199252. Correo electrónico: beatriz.chaverra@udea.edu.co

\section{Resumen}

Este estudio pretendió conocer e interpretar el discurso evaluativo de seis docentes de educación física durante la fase interactiva. La investigación asumió un diseño cualitativo a través del estudio de caso múltiple. Se grabaron en video y audio todas las sesiones de clase de una unidad didáctica y se utilizó un instrumento para la transcripción y análisis del discurso. En el análisis del discurso evaluativo surgieron nueve categorías. Se destacó la evaluación de las actitudes del estudiantado tanto positivas (motivación - felicitación) como negativas (censura-llamado de atención), relegando la información sobre las propias tareas motrices que aparecen en el tercer (correctiva) y cuarto lugar (ofrecimiento de soluciones). En los últimos puestos se ubica la información que le permitiría al estudiantado interiorizar el aprendizaje, ser conscientes de lo que están realizando y desarrollar su autonomía (argumentativa, interrogativa, indicación de errores).

Palabras clave: educación física; discurso evaluativo; evaluación formativa

\begin{abstract}
This study aimed to know and interpret the evaluative discourse of six physical education teachers during the interactive phase. The research assumed a qualitative design through the multiple case study. All the class sessions of a didactic unit were recorded in video and audio. In addition, it was used an instrument for the transcription and analysis of the discourse of teachers. Nine categories emerged in the analysis of evaluative discourse. The assessment of students' attitudes was positive (motivation congratulation) and negative (censorship-call for attention), relegating the information about the own motor tasks that appear in the third (corrective) and fourth place (offering Análisis del discurso evaluativo en docentes de educación física
\end{abstract}


of solutions). In the last positions is located information that would allow students to internalize learning, be aware of what they are doing and develop their autonomy (argumentative, interrogative, indication of errors).

Keywords: physical education; evaluative discourse; formative assessment

\section{Resumo}

Este estudo teve como objetivo compreender e interpretar o discurso avaliativo de seis professores de educação física durante a fase interativa. Demorou um projeto de pesquisa qualitativa por meio de estudo de caso múltiplo. Todas as sessões de classe duma unidade de ensino foram gravadas em vídeo e áudio, e foi utilizado um instrumento para a análise da transcrição e da fala. Em o discurso avaliativo surgiu nove categorias. Se destacou a avalição das atitudes positivas dos alunos (motivação felicitação) e negativas (censura- chamado a atenção), relegando informações sobre as próprias tarefas motoras que aparecem em terceiro (corretiva) e quarta (oferecendo soluções). Em último lugar esta a informação que permitiria a os alunos a internalizar a aprendizagem, estar ciente de que eles estão fazendo e desenvolver a sua autonomia (argumentação, interrogativa, indicação erros).

Palavras-chave: educação física; discurso avaliativo; avaliação formativa

\section{Introducción}

Múltiples estudios han analizado el discurso del profesorado en educación física (EF) con diferentes metodologías y enfoques (Giraldo, Rubio, \& Fernández, 2009; Hernández, 2002; Jiménez, Álvarez, Gómez, \& Guerra, 2003; López, 2012; Velázquez \& López, 2010; Webster, González, \& Harvey, 2012). Sin embargo, indagar por el discurso evaluativo es un aspecto reciente (Chaverra, 2017). Este texto presenta resultados parciales de una investigación sobre concepciones y acción evaluativa de seis docentes de EF en Medellín-Colombia. Específicamente se exponen los hallazgos sobre el objetivo: Conocer e interpretar el discurso evaluativo del profesorado de EF durante la fase interactiva de la enseñanza.

\section{Método}

El diseño fue cualitativo a través del estudio de caso colectivo (Stake, 2010). Participaron seis docentes: dos mujeres (Diana y María) y cuatro hombres (Juan, Carlos, 
Luis y Pedro). La investigadora grabó en video y audio todas las sesiones de clase de una unidad didáctica en cada docente (51 sesiones). La transcripción del discurso se realizó en un formato utilizado en estudios anteriores (López, 2012; Velázquez et al., 2007) con adaptaciones a esta investigación (Figura 1).

\begin{tabular}{|c|c|c|c|c|c|}
\hline \multicolumn{6}{|c|}{$\begin{array}{l}\text { Nombre de la profesora: Diana } \\
\text { Unidad didáctica: Gimnasia con } \\
\text { Clase } \mathbf{N}^{\mathbf{0}}: 1 \\
\text { Fecha: agosto } 21 \text { de } 2013 \\
\text { Duración: } 1 \mathrm{~h}: 31 \mathrm{~m}: 26 \mathrm{~s} \\
\end{array}$} \\
\hline Tiempo & $\begin{array}{l}\text { Desarrollo } \\
\text { de la sesión }\end{array}$ & $\begin{array}{c}\text { Discurso de la } \\
\text { profesora }\end{array}$ & Situación & $\begin{array}{c}\text { Tipo de } \\
\text { información } \\
\text { evaluativa }\end{array}$ & Observaciones \\
\hline 00h:20m:34s & Tarea 1 & $\begin{array}{l}\text { Muy bien, los veo } \\
\text { muy bien... }\end{array}$ & $\begin{array}{l}\text { Toda la } \\
\text { clase }\end{array}$ & $\begin{array}{l}\text { Motivación - } \\
\text { Felicitación }\end{array}$ & \\
\hline
\end{tabular}

Figura 1.

Instrumento para la transcripción y el análisis del discurso evaluativo.

Las categorías del discurso evaluativo emergieron de la lectura de la información y la literatura sobre el tema. Estas categorías se explican en la Figura 2.

\begin{tabular}{ll}
\hline \multicolumn{1}{c}{ CATEGORÍA } & \multicolumn{1}{c}{ DESCRIPCIÓN } \\
\hline Argumentativa & $\begin{array}{l}\text { Explica las razones por las que el estudiante está realizando bien } \\
\text { la tarea o está cometiendo un error. Puede dar ideas sobre cómo } \\
\text { hacerla mejor o preguntar al estudiante si tiene alternativas de } \\
\text { solución. }\end{array}$ \\
\hline Censura-Llamado de atención & $\begin{array}{l}\text { Tiene por objeto censurar la conducta sea por acción o por } \\
\text { omisión. }\end{array}$ \\
\hline Correctiva & Da indicaciones sobre cómo debe hacerse la tarea correctamente. \\
\hline Indicación de errores & $\begin{array}{l}\text { Señala el error cometido durante la tarea, pero no aporta ninguna } \\
\text { alternativa o explicación. }\end{array}$ \\
\hline Informativa & $\begin{array}{l}\text { Presenta los criterios de evaluación de la unidad didáctica, la } \\
\text { clase o la actividad. Asimismo, comunica cómo llevan las } \\
\text { calificaciones para aprobar o reprobar la unidad didáctica. }\end{array}$ \\
\hline Interrogativa & $\begin{array}{l}\text { Realiza preguntas para que verbalicen cómo consideran que fue } \\
\text { su desempeño durante la tarea o el de sus compañeros. }\end{array}$ \\
\hline Motivación-Felicitación & $\begin{array}{l}\text { Frases cortas o estímulos verbales para motivar al estudiantado a } \\
\text { continuar realizando la tarea o felicitarlos por su desempeño. }\end{array}$ \\
\hline Ofrecimiento de soluciones & Aporta una idea sobre cómo hacer mejor la tarea. \\
\hline Sancionadora & $\begin{array}{l}\text { Utiliza la calificación como una forma de controlar el trabajo en } \\
\text { clase. }\end{array}$ \\
\hline
\end{tabular}

Figura 2.

Categorías que emergieron en el análisis del discurso evaluativo del profesorado.

El tratamiento de la información se realizó a través del software Nvivo 10, permitiendo realizar una lectura que llevara a la refinación y depuración de la información (Taylor \& Bogdan, 1987), logrando identificar las categorías más frecuentes y/o ausentes en el profesorado. 


\section{Resultados y discusión}

En la Tabla 1 se muestran las frecuencias de las expresiones evaluativas del profesorado.

Tabla 1.

Frecuencia de categorías evaluativas en el discurso del profesorado

\begin{tabular}{lc}
\hline \multicolumn{1}{c}{ Subcategoría } & Frecuencia \\
\hline Motivación-Felicitación & 408 \\
Censura-Llamado de atención & 377 \\
Correctiva & 279 \\
Ofrecimiento de soluciones & 149 \\
Informativa & 131 \\
Argumentativa & 47 \\
Indicación de errores & 45 \\
Interrogativa & 30 \\
Sancionadora & 22 \\
\hline
\end{tabular}

Los resultados muestran que el discurso evaluativo se concentró en alabar y felicitar al estudiantado. Estos mensajes no se centraron en los aprendizajes de las tareas motrices, sino en el esfuerzo, la buena actitud y la participación durante las clases.

... todos se dan un aplauso que trabajaron muy bien. (C.2.J. $)^{1}$

El segundo lugar es ocupado por la evaluación de las actitudes disruptivas: La falta de escucha, el mal uso de los materiales de clase, no realizar las tareas, el uso de teléfonos móviles, etc.

...usted no ha hecho nada, yo estoy pendiente de usted. (C.2.M.)

En el tercer y cuarto lugar aparecen las categorías relacionadas con las tareas motrices. La información correctiva tuvo prevalencia sobre el ofrecimiento de soluciones, lo que indica que el profesorado optó por modificar directamente las acciones del estudiantado en lugar de brindar alternativas para que ellos encontraran la mejor manera de realizarlas.

En los últimos lugares aparecen las expresiones argumentativas, indicación de errores e interrogativas. Este resultado es un aspecto a mejorar si se tiene en cuenta que estas expresiones favorecen la reflexión sobre los aspectos centrales de la tarea realizada, brindan información sobre las dificultades presentadas y señalan los aciertos

\footnotetext{
${ }^{1}$ La codificación de los datos se realizó según la fuente. En este caso la sigla (C.2.J) significa clase 2 de Juan. 
que permitieron una adecuada realización. En definitiva, buscan que el estudiantado tome conciencia y dote de sentido los aprendizajes que se producen en la clase.

En el caso de las expresiones sancionatorias, puede asumirse como un aspecto positivo que estén poco presentes en el discurso. Sin embargo, son un reflejo de cómo la calificación sigue siendo un mecanismo de presión y control.

\section{Conclusiones}

El discurso evaluativo estuvo centrado fundamentalmente, en motivar y felicitar al estudiantado por su desempeño. Estas frases permiten un contacto permanente con el estudiantado, pero llegan a convertirse en un acto mecánico, que alienta y felicita, aunque se estén cometiendo errores. Asimismo, la alta frecuencia en expresiones de censura-llamado de atención señala un interés por mantener la disciplina de la clase.

Identificar estas categorías como las más relevantes, es admitir que se relega otro tipo de información valiosa, como presentarle al estudiantado por qué se están haciendo bien o mal las tareas, cómo se puede mejorar el desempeño, o preguntarles cómo podría hacerse mejor; información que les permitiría interiorizar el aprendizaje y ser conscientes de lo que están realizando.

Es posible que el profesorado no conciba la evaluación como una acción que se hace presente a través del discurso, de ahí que no sean conscientes del impacto que tienen sus expresiones en la enseñanza.

\section{Referencias}

Chaverra, B. (2017). Pensamiento y acción evaluativa del profesorado de educación física de educación secundaria y media: Estudio de casos en Medellín-Colombia. Tesis doctoral, Universidad Autónoma de Madrid, Madrid.

Giraldo, L., Rubio, E., \& Fernández, J. (2009). Caracterización del discurso pedagógico del docente de educación física e identificación de los actos de habla que estimulan la creatividad motriz. Ágora para la EF y el Deporte, 11, 25-41.

Hernández, J. (2002). Diseño y validación de un instrumento para el análisis del proceso de comunicación docente en torno a las tareas de enseñanza de la educación física. Tándem: Didáctica de la Educación Física, (9), 91-107. 
Jiménez, F., Álvarez, A., Gómez, A., \& Guerra, G. (2003). Iniciación deportiva y socialización en el ámbito escolar: análisis del discurso docente. En XXI Congreso Nacional de Educación Física “El pensamiento del profesorado” (pp. 1-17). Santa Cruz de Tenerife: Dirección General de Ordenación e Innovación Educativa del Gobierno de Canarias.

López, A. (2012). La calidad de los procesos de comunicación en el aula de Educación Física: un estudio sobre calidad del discurso docente en profesorado de la Comunidad de Madrid. Tesis Doctoral, Universidad Autónoma de Madrid, Madrid.

Stake, R. (2010). Investigación con estudio de casos (5ª). Madrid: Morata.

Taylor, S., \& Bogdan, R. (1987). Introducción a los métodos cualitativos de investigación. Barcelona: Paidós.

Velázquez, R., Hernández, J., Garoz, I., López, C., López, M., Maldonado, A., ... Castejón, J. (2007). Calidad de enseñanza en Educación Física y Deportiva y discurso docente: el caso de la Comunidad de Madrid. Revista de Educación, (344), 237-238.

Velázquez, R., \& López, A. (2010). El proceso de comunicación docente-discente en educación física. En J. Hernández \& R. Velázquez (Eds.), La educación física a estudio. El profesorado, el alumnado y los procesos de enseñanza (pp. 37-67). Barcelona: Graó.

Webster, C., González, S., \& Harvey, R. (2012). Physical Education Teachers ' SelfReported Communication of Content Relevance. The Physical Educator, 69(1), 89-103. 